\title{
Un phénomène de Hartogs dans les variétés projectives
}

\author{
Pascal Dingoyan
}

Received: 16 July 2007 / Accepted: 18 April 2008 / Published online: 26 June 2008 C Springer-Verlag 2008

\section{Erratum to: Math. Z. (1999) 232:217-240 DOI 10.1007/s002090050511}

Let $V$ be a projective manifold, $\operatorname{dim} V \geq 2$. Let $Z$ be an open subset in $V$ which is pseudoconcave (see [1]). Let $\mathcal{M}(Z)$ be the field of meromorphic functions on $Z$. According to Andreotti [1], $\mathcal{M}(Z)$ is a finite extension of $\mathcal{M}(V)$. Hence meromorphic functions on a pseudoconcave domain in a projective manifold are algebraic. In [3], Theorem 3.2.1, we claimed that they are rationals. This claim is false. Counterexamples can be found in [6], Chapter 5, page 199 or in [2], chapter 9, example 9.1.

In order to state a Hartogs' theorem on a projective manifold, we must take care of algebraic multivaluedness. We state corrected versions of main theorem 3.2.4 in [3] and of Corollary 6 of [4]. First, we work with a pseudoconcave domain $Z$ such that $\mathcal{M}(Z) \simeq \mathcal{M}(V)$. Then, we use a primitive element to reduce to the first case.

Theorem Let $V$ be a projective manifold, $\operatorname{dim} V \geq 2$. Let $U$ be an open subset in $V$ such that $V \backslash \bar{U}$ is pseudoconcave in the sense of Andreotti and the boundary of $U$ is connected. Let $H$ be the maximal compact reduced divisor in $U$ (see [3]). Assume meromorphic functions on $V \backslash \bar{U}$ are rationals. Let $F \rightarrow V$ be a holomorphic vector bundle. Then any meromorphic section of $F$ defined on a connected neighborhood $W$ of $\partial U$ extends as a meromorphic section of $F$ over $U$. Moreover if that section is holomorphic on $W$, then its extension is holomorphic on $U \backslash H$.

Proof In the proof of theorem 3.2.4 of [3] line 34, use the hypothesis that meromorphic functions on $V \backslash \bar{U}$ are rationals instead of using theorem 3.2.1.

The second corrected version works for general pseudoconcave domains.

The online version of the original article can be found under doi:10.1007/s002090050511.

P. Dingoyan $(\varangle)$

Faculté de Mathématiques, Université Pierre et Marie Curie,

175 rue du Chevaleret, 75013 Paris, France

e-mail: dingoyan@math.jussieu.fr 
Theorem Let $V$ be a projective manifold, $\operatorname{dim} V \geq 2$. Let $Z$ be a pseudoconcave domain in the sense of Andreotti with $\partial Z$ connected. Assume $U=V \backslash \bar{Z} \neq \emptyset$. Then there exists a branched covering $\Pi: X \rightarrow V$ with a section $S$ on $Z$ such that meromorphic functions on $Z$ are pull backs by $S$ of rational functions on $X$ (Theorem of Andreotti). Let $\tilde{U}=X \backslash \bar{S}(Z)$. Let $F \rightarrow V$ a holomorphic vector bundle. If $\alpha$ is a meromorphic section of $F$ over a connected neighborhood $W$ of $\partial Z$, then $\alpha$ is the pulback by $S_{\mid W}$ of a meromorphic section of $\Pi^{*}(F)$ defined on a neighborhood of $\tilde{U}$. Hence, $\mathcal{M}(W)$ is a finite algebraic extension of $\mathcal{M}(U)$.

Proof According to Andreotti's theorem (see [1]), $\mathcal{M}(Z)$ is a finite algebraic extension of $\mathcal{M}(V)$. Let $f \in \mathcal{M}(Z)$ be a primitive element. Let $\Gamma_{f} \subset V \times \mathbb{P}^{1}$ be its graph. It is contained in a unique irreducible algebraic set $V_{1} \subset V \times \mathbb{P}^{1}$ of the same dimension (for $f$ is algebraic). Let $\alpha_{1}: V_{2} \rightarrow V_{1}$ be the normalization and let $V_{2} \rightarrow X \rightarrow V$ be the Stein factorization of $\pi_{V} \circ \alpha_{1}$ (with $\pi_{V}: V \times \mathbb{P}^{1} \rightarrow V$ the canonical projection). Then $X$ is normal (for $V_{2}$ is) and $\pi: X \rightarrow V$ is a finite map. Let $I_{f} \subset Z$ be the indeterminacy of $f$. Then $f$ determines a section of $\pi$ over $Z \backslash I_{f}$ (a normal space). Since $V$ is normal and $\operatorname{codim} I_{f}=2$, this section extends through $I_{f}$ as a section $S: Z \rightarrow X$ of $\Pi$. Hence $X$ is smooth along this section. Let $\mu: \tilde{X} \rightarrow X$ be a desingularisation of $X$ which is biholomorphic on $S(Z)$. Then $\tilde{X}$ is projective and $\mu^{-1} \circ S(Z)$ is a pseudoconcave domain whose meromorphic functions are rationals. Let $F \rightarrow V$ be a vector bundle and let $s$ be a meromorphic section of $F$ over $W$. Let $W^{\prime}$ be the connected component of $(\mu \circ \Pi)^{-1}(W)$ which contains $\left(\mu^{-1} \circ S\right)(Z \cap W)$. Then $W^{\prime}$ is a neighborhood of $\partial \tilde{U}$. Moreover, $\left(\mu^{-1} \circ S\right)_{*}(s \mid W \cap Z)$ extends as $(\pi \circ \mu)^{*}(s)_{\mid W^{\prime}}$, a section of $(\pi \circ \mu)^{*}(F)$. We apply the first theorem to this data on $\tilde{X}$. Since $\mu$ is biholomorphic outside an analytic set of codimension at least two, the first assertion is proved. Since $\Pi^{-1}(U) \subset \tilde{U}$ and $\Pi_{\mid \Pi^{-1}(U)}: \Pi^{-1}(U) \rightarrow U$ is finite and proper, the second assertion is proved.

Addendum. In the proof of Proposition 3.1.3 of [3], we assumed $C$ irreducible. However, the proof works for any effective divisor with pseudoconcave complement. Hence Grauert's criterion for contractibility [5] implies that the support of the divisor with pseudoconcave complement may be blown down as claimed.

\section{References}

1. Andreotti, A.: Théorème de dépendance algébrique sur les espaces complexes pseudo-concaves. Bull. Soc. Math. France 91, 1-38 (1963)

2. Bădescu, L.: Projective geometry and formal geometry, Mathematics Institute of the polish Academy of Sciences. Mathematical Monographs (New Series), 65. Birkhauser-Verlag (2004)

3. Dingoyan P.: Un phénomène de Hartogs dans les variétés projectives. Math. Zeit. 232, 217-240 (1999)

4. Dingoyan, P.: Monge-Ampère currents over pseudoconcave spaces Math. Ann. 320, 211-238 (2001)

5. Grauert, H.: Über Modifikationen und exzeptionelle analytische Mengen. Math. Ann. 146, 331-368 (1962)

6. Hartshorne, R.: Ample Subvarieties of algebraic varieties. In: Lecture Notes in Math., vol. 156. SpringerVerlag (2004) 